\title{
ON THE REPRESENTATIONS OF AN INTEGER AS THE SUM OF PRODUCTS OF INTEGERS
}

\author{
BY \\ S. M. JOHNSON
}

1. Introduction $\left({ }^{1}\right)$. The problem of finding the number of representations of an integer $N$ as a sum of products of pairs of positive integers was first studied by Ingham and Estermann. By elementary methods Ingham [5](2) found an asymptotic formula for the number $R(N)$ of representations of $N$ as a sum of two products of pairs. His result was $R(N)=(1 / 2 \zeta(2)) \cdot \sigma(N) \log ^{2} N$ $+O(\sigma(N) \log N)$ where $\sigma(N)$ is the sum of the divisors of $N$.

Estermann $[2 ; 3]$, using Hardy and Littlewood's circle method of analytic number theory, found a more precise asymptotic formula for the above problem and also for the case of representing $N$ as a sum of three products of pairs of positive integers. The error terms were smaller than the leading terms by an order of a fractional power of $N$.

Equivalent forms for these expressions are

$$
\sum_{a+b=N} d(a) d(b), a, b>0, \quad \text { and } \sum_{a+b+c=N} d(a) d(b) d(c), a, b, c>0 .
$$

A similar type of problem involving sums of the form $\sum_{n \leqq N} d(n) d(n+b)$, $b$ fixed, has been solved asymptotically by Ingham [5] and later by Estermann [3]. Bellman [1] studied $\sum_{n \leqq N} d(n) d_{3}(n+b)$ and $\sum_{n \leqq N} d(n) d_{4}(n+b)$. Titchmarsh [7] used the circle method to obtain heuristic results for various problems of these types involving triple products. He studied the conjecture that correct results could be obtained in a number of problems by proceeding in the same way as for products of pairs. No rigorous techniques are available at this time to handle the error terms. His conclusion was that the conjecture is false in the case of $\sum_{n \leqq N} d_{3}^{2}(n)$. The heuristic result did not agree with the result given by elementary methods.

In the present paper we solve a particular problem involving products of triples by a modification of the circle method considerably different from that used by Estermann and Titchmarsh. We obtain an asymptotic formula for the number of representations of an integer $N$ as a sum of three products of triples and show how the method may be generalized to the case where $N$ is represented as a sum of $k n$-tuple products $(k \geqq 3, n \geqq 2)$. A further gen-

Received by the editors November 26, 1952.

(1) The results of this paper are contained in a thesis submitted in partial requirement for a Ph.D. degree granted in 1950 at the University of Illinois. The author wishes to express his gratitude to Professor L. K. Hua for his guidance in this problem.

(2) Numbers in brackets refer to the bibliography at the end of the paper. 
eralization leads to the case where the $j$ th product term has $n_{j}$ factors. For $k=2$ the method used here fails to handle the minor arc error terms.

Only the leading term is obtained with error terms smaller by an order of $1 / \log N$. More precise results for some of the error terms could be found, but this would not help the final result in view of Lemma 3. In the special case for products of pairs our result checks with the leading term of Estermann's results.

2. Analytic formulation and Farey dissection. We first consider the problem of representing $N$ as a sum of three triple products. Subsequently we shall show how the results may be generalized to $k$ products where $k \geqq 3$ and $n_{j} \geqq 2$, $j=1,2, \cdots, k$.

Let $R(N)$ be the number of solutions of $N=x_{11} x_{12} x_{13}+x_{21} x_{22} x_{23}+x_{31} x_{32} x_{33}$ where the $x$ 's are positive integers. Our fundamental generating function will be

$$
S(t)=\sum_{x_{1} x_{2} x_{3} \leqq N} e^{2 \pi i x_{1} x_{2} x_{3} t}, \quad x_{j}>0, j=1,2,3 .
$$

This technique of using trigonometric polynomials in place of power series is due to Vinogradoff. It obviates many difficulties of convergence, analytic behavior, etc. which are present in the original circle method of Hardy, Littlewood, and Ramanujan.

We note that

$$
R(N)=\int_{0}^{1} S^{3}(t) e^{-2 \pi i N t} d t .
$$

We now begin the Farey dissection. One must divide the unit circle into a collection of so-called major arcs and minor arcs in such a way that the integration in (1) over the major arcs gives the correct leading term in the asymptotic formula. This is done by a suitable choice of parameters defining these arcs, which can be obtained only by a certain amount of trial and error and experience. In our case we let $P=N^{1 / 3}$ and $Q=N^{11 / 12}$. Then

$$
R(N)=\int_{-1 / Q}^{1-1 / Q} S^{3}(t) e^{-2 \pi i N t} d t
$$

since the integrand has period one.

Consider the set of $h$ and $q$ such that $1 \leqq h \leqq q \leqq Q$ with $(h, q)=1$. Let $M_{h q}$ be the set of all $t$ such that $|t-h / q| \leqq 1 / q Q$ and $-1 / Q \leqq t \leqq 1-1 / Q$, where $1 \leqq q \leqq N^{1 / 12}=P^{1 / 4}$. It is easily seen that no two sets $M_{h q}$ overlap. Let $E$ be the set of all $t$ in $(-1 / Q, 1-1 / Q)$ but not in any $M_{h q}$. Then

$$
R(N)=\left(\sum_{1 \leqq q \leqq P^{1 / 4}} \sum_{1 \leqq h \leqq q,(h, q)=1} \int_{M h q}+\int_{E}\right) S^{3}(t) e^{-2 \pi i t N} d t .
$$

It will be shown in the next section that the contribution to $R(N)$ from the 
set $E$ is of smaller order of magnitude than that from the set of major arcs $M_{h q}$.

3. The minor arcs. In order to obtain our estimate we use the obvious inequality

$$
\begin{aligned}
\int_{E} S^{3}(t) e^{-2 \pi i N} t d t & <\left(\max _{t \text { in } E}|S(t)|\right) \int_{E}|S(t)|^{2} d t \\
& <\left(\max _{t \text { in } E}|S(t)|\right) \int_{0}^{1}|S(t)|^{2} d t .
\end{aligned}
$$

For subsequent use we require

$$
\int_{0}^{1}|S(t)|^{2} d t=\sum_{m \leqq N}\left(d_{3}(m)\right)^{2}=O\left(N^{1+\epsilon}\right), \quad \epsilon>0 .
$$

Lemma 1. Let $C_{0}$ be the set of conditions $1 \leqq x_{j} \leqq P, j=1,2,3$, and $C_{1}$ the set of conditions $x_{1} x_{2} x_{3} \leqq N, x_{1} x_{2} \leqq P^{2}, x_{1} \leqq P$. Let

$$
S_{0}(t)=\sum_{C_{0}} e^{2 \pi i x_{1} x_{2} x_{3} t}, \quad S_{1}(t)=\sum_{C_{1}} e^{2 \pi i x_{1} x_{2} x_{3} t} .
$$

Then $S(t)=S_{0}(t)+3 S_{1}(t)$.

Proof. This is merely a special case of a general combinatorial theorem (Hardy [4, cf. p. 232]). Let $M$ be the total set of elements each of which may or may not satisfy one or more of the conditions $A_{1}, \cdots, A_{m}$. Let $M\left(A_{1}, A_{2}\right)$ be the set of elements satisfying $\left(A_{1}, A_{2}\right)$, etc. Then the theorem states that a decomposition of the set of elements not satisfying any of these conditions is given by

$$
M-\sum_{j} M\left(A_{j}\right)+\sum_{j, k ; j \neq k} M\left(A_{j}, A_{k}\right) \cdots(-1)^{m} M\left(A_{1}, \cdots, A_{m}\right) .
$$

Applying this result to our problem, let $A_{j}$ be the condition that $x_{j}>P$. Here we have

$$
\begin{aligned}
\sum_{x_{j} \leqq P, j=1,2,3} 1= & \sum_{x_{1} x_{2} x_{3} \leqq N} 1-3 \sum_{x_{1} x_{2} x_{3} \leqq N, x_{3}>P} 1 \\
& +3 \sum_{x_{1} x_{2} x_{3} \leqq N, x_{2}>P, x_{3}>P} 1-\sum_{x_{1} x_{2} x_{3} \leqq N, x_{j}>P, j=1,2,3} 1 .
\end{aligned}
$$

The last summation on the right is empty. After combining the next two summations and solving for the first we obtain the desired decomposition. This applied to the exponential sum yields Lemma 1.

LEMMA 2.

$$
\max _{t \text { on } E}|S(t)|=O\left(N^{11 / 12+\bullet}\right), \quad \quad \epsilon>0 .
$$


Proof. We have

$$
\begin{aligned}
& \left|S_{0}(t)\right| \leqq \sum_{x_{1} \leqq P, x_{2} \leqq P}\left|\sum_{x_{3} \leqq P} e^{2 \pi i x_{1} x_{2} x_{3} t}\right|, \\
& \left|S_{1}(t)\right| \leqq \sum_{x_{1} x_{2} \leqq P^{2}}\left|\sum_{P \leqq x_{3} \leqq N / x_{1} x_{2}} e^{2 \pi i x_{1} x_{2} x_{3} t}\right| .
\end{aligned}
$$

Let $x_{1} x_{2}=a$. Then we can write

$$
|S(t)| \leqq 4 \sum_{a \leqq P^{2}} d_{2}(a)\left(\left|\sum_{x_{3} \leqq N / a} e^{2 \pi i a t x_{3}}\right|+\left|\sum_{x_{3} \leqq P} e^{2 \pi i a t x_{3}}\right|\right) .
$$

For real $A$ let $\langle A\rangle$ be the distance from $A$ to the nearest integer. If $\min (L, 1 / 2\langle 0\rangle)$ is understood to be $L$, it follows from a well known result (Landau [6, cf. p. 253]) that

$$
\begin{aligned}
& \left|\sum_{x_{3} \leqq N / a} e^{2 \pi i a t x_{3}}\right| \leqq \min \left(\frac{N}{a}, \frac{1}{2\langle a t\rangle}\right) \text { and } \\
& \left|\sum_{x_{3} \leqq P} e^{2 \pi i a t x_{3}}\right| \leqq \min \left(P, \frac{1}{2\langle a t\rangle}\right) .
\end{aligned}
$$

Therefore

$$
\begin{aligned}
|S(t)| & \leqq 4 \sum_{a \leqq P^{2}} d_{2}(a)\left\{\min \left(\frac{N}{a}, \frac{1}{2\langle a t\rangle}\right)+\min \left(P, \frac{1}{2\langle a t\rangle}\right)\right\} \\
& \leqq 4 \sum_{a \leqq P^{2}} d_{2}(a) \min \left(\frac{N}{a}, \frac{1}{2\langle a t\rangle}\right)
\end{aligned}
$$

since $N / a \geqq P$.

It is known (Hardy [4, cf. Theorem 36]) that for $t$ in the range $-1 / Q$ $\leqq t \leqq 1-1 / Q$, there is some pair of integers $h, q$ such that $1 \leqq h \leqq q \leqq Q$, $(h, q)=1$, and $|t-h / q| \leqq 1 / q Q$.

On set $E$ we have $P^{1 / 4}<q \leqq Q=P^{11 / 4}$. Therefore we can write $t a=h a / q$ $+r a / q Q$ where $|r| \leqq 1$. Let $h a \equiv b(\bmod q)$. Then $\langle a t\rangle=\langle b / q+r a / q Q\rangle$. As $a$ runs through its values $1,2, \cdots,\left[P^{2}\right]$, let $b$ be chosen so that $-q / 2<b \leqq q / 2$. Each $b$ appears at most $P^{2} / q+1$ times. When $b=0$, choose $N / a=N / m q$; otherwise choose $1 / 2\langle b / q+r a / q Q\rangle$. We have $\langle b / q+r a / q Q\rangle \geqq\langle|b| / q-a / q Q\rangle$ $\geqq\left\langle\left(|b|-P^{-3 / 4}\right) / q\right\rangle=\left(|b|-P^{-3 / 4}\right) / q$ since $|b| / q \leqq 1 / 2$ and $|b|-P^{-3 / 4}<q / 2$. Thus

$$
\sum_{a \leqq P^{2}} \min \left(\frac{N}{a}, \frac{1}{2\langle a t\rangle}\right)<\sum_{1 \leqq m \leqq P^{2} / q} \frac{N}{m q}+2\left(\frac{P^{2}}{q}+1\right) \sum_{1 \leqq b \leqq q / 2} \frac{q}{b-P^{-3 / 4}} .
$$

Next, $\sum_{1 \leqq m \leqq P^{2} / q} N / m q=O((N / q) \log N)=O\left(P^{11 / 4} \log N\right)$ since $q>P^{1 / 4}$. Also, 
$\left(P^{2}+q\right) \sum_{1 \leqq b \leqq q / 2} 1 /\left(b-P^{-3 / 4}\right)=O\left(\left(P^{2}+q\right) \quad \log N\right)=O\left(P^{11 / 4} \log N\right)$ since $q \leqq P^{11 / 4}$. Thus $|S(t)|=O\left(N^{11 / 12+\epsilon}\right)$ for $t$ on $E$ since $\log N=O\left(N^{\epsilon / 2}\right)$ and $d_{2}(a)=O\left(N^{\epsilon / 2}\right)$ for $\epsilon>0$. This completes the proof of Lemma 2 .

Finally by (1), (2), and (4) we have

ThEOREM 1.

$$
\int_{E} S^{3}(t) e^{-2 \pi i N} t d t=O\left(N^{23 / 12+\epsilon}\right) .
$$

4. The major arcs. In this section we calculate the contribution of the major arcs to the integral expression for $R(N)$.

By definition, $M_{h q}$ is the set of $t=h / q+g$ where $|g| \leqq 1 / q Q, 1 \leqq q \leqq P^{1 / 4}$, $Q=P^{11 / 4}$. In order to evaluate $S(t)$ more precisely on a given $M_{h q}$, we let $x_{j}=y_{j}+q z_{j}$ where $1 \leqq y_{j} \leqq q, z_{j} \geqq 0, j=1,2,3$, and sum over the $y^{\prime}$ 's and $z$ 's separately subject to the conditions on the $x$ 's. Then for $m=0,1$ we can write

$$
S_{m}(t)=\sum_{1 \leqq y_{j} \leqq q, j=1,2,3} e^{2 \pi i(h / q) y_{1} y_{2} y_{3}} \sum_{z_{1}, z_{2}, z_{3}, C_{m}} e^{2 \pi i g x_{1} x_{2}\left(y 3+q z_{3}\right)}
$$

where $C_{0}=$ the set of conditions $y_{j}+q z_{j} \leqq P, j=1,2,3$, and $C_{1}=$ the set of conditions $\prod_{j=1}^{3}\left(y_{j}+q z_{j}\right) \leqq N, \prod_{j=1}^{2}\left(y_{j}+q z_{j}\right) \leqq P^{2}, y_{1}+q z_{1} \leqq P$.

First, sum over $z_{3}$ and $y_{3}$ in order to obtain more manageable sums. To do this we need several results $(2), \cdots,(6)$ which may be readily demonstrated.

We note that $|g a q| \leqq 1 / P^{3 / 4}<1 / 2$. Then if $g a q \neq 0$ and $1 \leqq a \leqq P^{2}$, and if $z_{3}$ is summed subject to $P<y_{3}+q z_{3} \leqq\left[N^{2} / a\right]$, we have

$$
\sum_{z_{3}} e^{2 \pi i g a\left(y 3+q z_{3}\right)}=\frac{e^{2 \pi i g N}-e^{2 \pi i g a P}}{2 \pi i g a q}+O(1) .
$$

Also, if $z_{3}$ is summed subject to $0<y_{3}+q z_{3} \leqq P$ we have

$$
\sum_{z_{3}} e^{2 \pi i g a\left(y_{3}+q z_{3}\right)}=\frac{e^{2 \pi i g a P}-1}{2 \pi i g a q}+O(1) .
$$

These results follow from summation of geometric series and routine error term estimates where we make use of the fact that $\mid$ gaq $\mid \leqq 1 / P^{3 / 4}<1 / 2$.

Defining $v(q)=\sum_{1 \leqq y_{1}, y_{2} \leqq q_{;} q \mid y_{1} y_{2}} 1$, we have, if $(h, q)=1$, that

$$
\sum_{1 \leqq y_{j} \leqq q, j=1,2,3} e^{2 \pi i(h / q) y_{1} y_{2 y} y_{3}}=q v(q) .
$$

Later we shall evaluate $v(q)$ explicitly for use in the section on the singular series.

Also, if

$$
F(q)=\sum_{1 \leqq y_{1}, y_{2} \leqq q ; q \mid y_{1} y_{2}} \frac{1}{y_{1} y_{2}}, \text { then } F(q)=O\left(\frac{1}{q^{1-\epsilon}}\right), \quad \epsilon>0 .
$$


Next if we sum over $y_{1}, y_{2}, z_{1}, z_{2}$, subject to $\left(y_{1}+q z_{1}\right)\left(y_{2}+q z_{2}\right) \leqq P^{2}$, we have

$$
\sum_{y_{1}, y_{2}, z_{1}, z_{2}} 1=O\left(P^{2} \log N\right) .
$$

After summing over $z_{3}$ and $y_{3}$ in (1), it follows from (2), (3), (4), (5), and (6) that

$$
S_{0}(t)=\sum \frac{e^{2 \pi i g a P}-1}{2 \pi i g a}+O\left(q P^{2} \log N\right)
$$

where the summation is over $z_{1}, z_{2}, y_{1}, y_{2}$ subject to $y_{1}+q z_{1} \leqq P, y_{2}+q z_{2} \leqq P$, and $q \mid y_{1} y_{2}$. Also

$$
S_{1}(t)=\sum \frac{e^{2 \pi i g N}-e^{2 \pi i g a P}}{2 \pi i g a}+O\left(q P^{2} \log N\right)
$$

where the summation is over $z_{1}, z_{2}, y_{1}, y_{2}$ subject to $\left(y_{1}+q z_{1}\right)\left(y_{2}+q z_{2}\right) \leqq P^{2}$, $y_{1}+q z_{1} \leqq P$, and $q \mid y_{1} y_{2}$.

Then let

$$
T_{1}=\sum^{\prime} \frac{e^{2 \pi i 0 N}-1}{2 \pi i g a}
$$

where $\sum$ indicates three times the summation over $z_{1}, z_{2}, y_{1}, y_{2}$, subject to the set of conditions $\left(y_{1}+q z_{1}\right)\left(y_{2}+q z_{2}\right) \leqq P^{2}, y_{1}+q z_{1} \leqq P$, and $q \mid y_{1} y_{2}$, and let

$$
T_{2}=\sum^{\prime \prime} \frac{e^{2 \pi i g a P}-1}{2 \pi i g a}
$$

where $\sum^{\prime \prime}=\sum_{0}-\sum^{\prime}$, and $\sum_{0}$ indicates summation over $z_{1}, z_{2}, y_{1}, y_{2}$ subject to the set of conditions $y_{1}+q z_{1} \leqq P, y_{2}+q z_{2} \leqq P$ and $q \mid y_{1} y_{2}$. Later we show that terms involving $T_{1}$ dominate the contribution to our final result.

Thus we have

$$
S(t)=T_{1}+T_{2}+O\left(q P^{2} \log N\right) .
$$

Next note that summing over $z$ subject to $z+y / q \leqq[N / q]$, we have

$$
\sum \frac{1}{z+y / q}=O\left(\frac{q}{y} \log N\right)
$$

so that by (5) it follows that

$$
\sum^{\prime} \frac{1}{a}=O\left(\frac{\log ^{2} N}{q^{1-c}}\right) .
$$

The same result holds for $\sum^{\prime \prime} 1 / a$.

Then by (12) we have 


$$
\left|T_{1}\right|=\left|\frac{e^{2 \pi i g N}-1}{2 \pi \imath g} \sum^{\prime} \frac{1}{a}\right|=O\left(\frac{1}{|g|} \frac{\log ^{2} N}{q^{1-\imath}}\right) .
$$

Similarly, we obtain a like estimate for $\left|T_{2}\right|$.

For future reference we need the following result:

$$
\text { Both } \sum^{\prime} 1 \text { and } \sum^{\prime \prime} 1 \text { are } o\left(\frac{P^{2} \log N}{q^{1-\epsilon}}\right) \text {. }
$$

To establish this we sum over $z_{2}$ subject to $z_{2}+y_{2} / q \leqq P^{2} / q^{2}\left(z_{1}+y_{1} / q\right)$. Then $\sum_{z_{2}} 1=P^{2} / q^{2}\left(z_{1}+y_{1} / q\right)$. Summing this over $z_{1}$, we obtain $O\left(\left(P^{2} / q y_{1}\right)\right.$ $\cdot \log N)=O\left(P^{2} \log N / y_{1} y_{2}\right)$. Then summing over $y_{1}, y_{2}$ subject to $q \mid y_{1} y_{2},(5)$ gives(14).

Next, let $H_{h q}=\int_{M_{h q}} S^{3}(t) e^{-2 \pi i t N} d t$.

We have $|S(t)| \leqq \sum_{x_{1} x_{2} x_{3} \leqq N} 1=O\left(N \log ^{2} N\right)$. Thus by (11) we also have $T_{1}+$ $T_{2}=O\left(N \log ^{2} N\right)$ since $q \leqq P^{1 / 4}<P$. Therefore $S^{3}(t)=\left(T_{1}+T_{2}\right)^{3}+O\left(P^{8} q \log ^{5} N\right)$.

It follows that

$$
\begin{aligned}
& \sum_{1 \leqq h \leqq q \leqq P^{1 / 4},(h, q)=1} H_{h q} \\
& =\sum_{q \leqq P^{1 / 4}} \sum_{\begin{array}{c}
1 \leqq h \leqq q,(h, q)=1 \\
\end{array}} e^{-2 \pi i(h / q) N} \int_{-1 / q Q}^{1 / q Q}\left(T_{1}+T_{2}\right)^{3} e^{-2 \pi i \sigma N} d g \\
& \quad+O\left(N^{23 / 12} \log ^{5} N\right) .
\end{aligned}
$$

Let us now show that the range of integration for $H_{h q}$ can be extended to $(-\infty,+\infty)$ with an error term in $H_{h q}$ of $O\left(N^{23 / 12+\epsilon}\right)$ for each $\epsilon>0$.

We have by (13)

$$
\int_{1 / Q Q}^{\infty}\left(T_{1}+T_{2}\right)^{3} e^{-2 \pi i 0 N} d g=O\left(\int_{1 / Q Q}^{\infty}\left(\frac{\log ^{2} N}{g q^{1-e^{\prime}}}\right)^{8} d g\right)=O\left(\frac{Q^{2} \log ^{6} N}{q^{1-3 \epsilon^{\prime}}}\right) .
$$

Therefore, summing this over $h$ and $q$ gives $O\left(N^{23 / 12+e}\right)$, since

$$
O\left(\sum_{q \leqq P^{1 / 4}} \sum_{h \leqq q} \frac{Q^{2} \log ^{6} N}{q^{1-3 \epsilon^{\prime}}}\right)=O\left(N^{11 / 6} \log ^{6} N \sum_{q \leqq P^{1 / 4}} q^{3 \epsilon^{\prime}}\right)=O\left(N^{23 / 12+\epsilon}\right) .
$$

We have as a result

$$
\begin{aligned}
R(N)= & \sum_{1 \leqq q \leqq p^{1 / 4}} \sum_{1 \leqq h \leqq q,(h, q)=1} e^{-2 \pi i(h / q) N} \int_{-\infty}^{\infty}\left(T_{1}+T_{2}\right)^{8} e^{-2 \pi i g N} d g \\
& +O\left(N^{28 / 12+e}\right) .
\end{aligned}
$$

Next we require

$$
\int_{-\infty}^{\infty}\left(\frac{e^{2 \pi i \sigma N}-1}{2 \pi i g}\right)^{8} e^{-2 \pi i \sigma N} d g=\frac{N^{2}}{2}
$$


After integrating by parts twice we obtain

$$
\frac{1}{2} \int_{-\infty}^{\infty}\left(\frac{4 N^{2} e^{4 \pi i g N}-3 N^{2} e^{2 \pi i g N}-N^{2} e^{-2 \pi i g N}}{2 \pi i g}\right) d g=\frac{N^{2}}{2}
$$

since by a well known result

$$
\int_{-\infty}^{\infty} \frac{e^{-i A g}}{g} d g=\pi i(\operatorname{sgn} A)
$$

where sgn $A=1,0,-1$ if $A$ is positive, zero, or negative respectively.

Expanding the integrand in (16) we get

$$
\int_{-\infty}^{\infty}\left(T_{1}+T_{2}\right)^{3} e^{-2 \pi i \sigma N} d g=I_{0}+3 I_{1}+3 I_{2}+I_{3}
$$

where $I_{m}=\int_{-\infty}^{\infty} T_{1}^{3-m} T_{2}^{m} e^{-2 \pi i \sigma N} d g, m=0,1,2,3$. Then

$$
I_{0}=\left(\sum^{\prime} \frac{1}{a}\right)^{3} \int_{-\infty}^{\infty}\left(\frac{e^{2 \pi i g N}-1}{2 \pi i g}\right)^{3} e^{-2 \pi i \sigma N} d g=\left(\sum^{\prime} \frac{1}{a}\right)^{3} \frac{N^{2}}{2} .
$$

Later we show $I_{0}$ leads to the dominant term in $R(N)$.

LEMMA 3. $I_{1}, I_{2}, I_{3}$ are complicated arithmetical expressions whose contributions to $R(N)$ are $O\left(N^{2} \log ^{5} N\right)$.

Proof. First consider $I_{1}$. Noting that the $a$ 's are to be distinguished by subscript here, we can write

$$
\left.I_{1}=\left(\sum^{\prime} \frac{1}{a_{1}}\right)\left(\sum^{\prime} \frac{1}{a_{2}}\right) \sum^{\prime \prime} \frac{1}{a_{3}}\right) \int_{-\infty}^{\infty} \frac{\left(e^{2 \pi i g N}-1\right)^{2}\left(e^{2 \pi i g P a_{3}}-1\right)}{(2 \pi i g)^{3}} e^{-2 \pi i g N} d g
$$

where the integral (after integrating by parts as for $\left.I_{0}\right)$ becomes $O\left(\mathrm{NPa}_{3}\right)$ since the terms involving $N^{2}$ cancel out. Thus by (12) and (14)

$$
I_{1}=O\left(N P\left(\sum^{\prime} \frac{1}{a_{1}}\right)\left(\sum^{\prime} \frac{1}{a_{2}}\right)\left(\sum^{\prime \prime} 1\right)\right)=O\left(\frac{N^{2} \log ^{5} N}{q^{3-\epsilon}}\right) .
$$

Summing over $h$ and $q$ gives the contribution to $R(N)$ of

$$
O\left(\sum_{q \leqq P^{1 / 4}} \frac{1}{q^{2-\epsilon}} N^{2} \log ^{5} N\right)=O\left(N^{2} \log ^{5} N\right) .
$$

We claim both $I_{2}$ and $I_{3}$ are also $O\left(\left(N^{2} \log ^{5} N\right) / q^{3-6}\right)$. For we have

$$
\begin{aligned}
I_{2}=\left(\sum^{\prime} \frac{1}{a_{1}}\right)\left(\sum^{\prime \prime} \frac{1}{a_{2}}\right) & \left(\sum^{\prime \prime} \frac{1}{a_{3}}\right) \\
& \cdot \int_{-\infty}^{\infty} \frac{\left(e^{2 \pi i g N}-1\right)\left(e^{2 \pi i g P a_{2}}-1\right)\left(e^{2 \pi i g P a_{3}}-1\right) e^{-2 \pi i g N} d g}{(2 \pi i g)^{3}}
\end{aligned}
$$


where the integral (after integrating by parts twice) equals

$$
\begin{array}{r}
\left(-N+P a_{2}+P a_{3}\right)^{2} \operatorname{sgn}\left(-N+P a_{2}+P a_{3}\right)-\left(-N+P a_{2}\right)^{2} \operatorname{sgn}\left(-N+P a_{2}\right) \\
-\left(-N+P a_{3}\right)^{2} \operatorname{sgn}\left(-N+P a_{3}\right)+(-N)^{2} \operatorname{sgn}(-N) .
\end{array}
$$

Notice that the sign of $-N+P a_{2}+P a_{3}$ can be + or - .

CASE 1. $a_{2}+a_{3} \geqq P^{2}$. Here the integral becomes

$\left(-N+P a_{2}+P a_{3}\right)^{2}+\left(-N+P a_{2}\right)^{2}+\left(-N+P a_{3}\right)^{2}-(-N)^{2}=O\left(N^{2}\right)$

since $0<\left(-N+P a_{2}+P a_{3}\right) \leqq N$. However $O\left(N^{2}\right) \leqq O\left(N\left(P a_{2}+P a_{3}\right)\right)$ since $P a_{2}$ $+P a_{3}>N$. Then

$$
\left.\left(\sum^{\prime \prime} \frac{1}{a_{2}}\right)\left(\sum^{\prime \prime} \frac{1}{a_{3}}\right)\left(P a_{2}+P a_{3}\right)=O\left(P\left(\sum^{\prime \prime} \frac{1}{a_{2}}\right)\left(\sum^{\prime \prime} 1\right)\right)\right)
$$

and therefore in this case $I_{2}$ is also $O\left(N^{2} \log ^{5} N / q^{3-\epsilon}\right)$.

CASE 2. $a_{2}+a_{3}<P^{2}$. Here the integral becomes

$$
-\left(-N+P a_{2}+P a_{3}\right)^{2}+\left(-N+P a_{2}\right)^{2}+\left(-N+P a_{3}\right)^{2}-(-N)^{2}
$$

which is $O\left(N P a_{2}\right)$ so that in this case $I_{2}$ is also $O\left(N^{2} \log ^{5} N / q^{3-\epsilon}\right) . I_{3}$ can be handled in the same way. This proves Lemma 3.

Thus we have

\section{THEOREM 2.}

$$
R(N)=\sum_{q \leqq P^{1 / 4}} \sum_{1 \leqq h \leqq q,(h, q)=1} e^{-2 \pi i(h / q) N}\left(\sum^{\prime} \frac{1}{a}\right)^{3} \frac{N^{2}}{2}+O\left(N^{2} \log ^{5} N\right) .
$$

Using the Euler-MacLaurin enumeration formula to evaluate $\sum^{\prime} 1 / a$, we have

$$
\sum_{z_{1}, z_{2}}^{\prime} \frac{1}{\left(z_{1}+y_{1} / q\right)\left(z_{2}+y_{2} / q\right)}+\frac{\log ^{2} N}{2}+O\left(\frac{q^{2} \log ^{2}(q+1) \log N}{y_{1} y_{2}}\right) .
$$

Finally, summing over $y_{1}, y_{2}$, by (5), we obtain

$$
\sum^{\prime} \frac{1}{x_{1} x_{2}}=\frac{v(q)}{q^{2}} \frac{\log ^{2} N}{2}+O\left(\frac{\log N}{q^{1-\epsilon}}\right) .
$$

This leads to

THEOREM 3.

$$
R(N)=\sum_{q=1}^{\infty} \sum_{1 \leqq h \leqq q,(h, q)=1} e^{-2 \pi i(h / q) N}\left(\frac{v(q)}{q^{2}}\right)^{3} \frac{N^{2} \log ^{6}}{2 !}+O\left(N^{2} \log ^{5} N\right) .
$$

The sum over $q$ can be extended to $+\infty$ since 


$$
\sum_{Q>P^{1 / 4}} q\left(\frac{1}{q^{1-\epsilon^{\prime}}}\right)^{3}=\sum_{Q>P^{1 / 4}} \frac{1}{q^{2-\epsilon^{\prime}}}=O\left(P^{-1 / 4+\epsilon}\right) .
$$

5. The singular series. We wish to evaluate

$$
S(N)=\sum_{q=1}^{\infty} \sum_{1 \leqq h \leqq q,(h, q)=1} e^{-2 \pi i(h / q) N}\left(\frac{v^{8}(q)}{q^{6}}\right) .
$$

By (4) of $\S 4, v(q)$ is the number of solutions $\left(y_{1}, y_{2}\right)$ of $y_{1} y_{2} \equiv 0(\bmod q)$ where $1 \leqq y_{1}, y_{2} \leqq q . v(q)$ has the following properties:

(a) $v(q)=\sum_{1 \leqq y \leqq q}(y, q)$, where $(y, q)=$ the g.c.d. of $y$ and $q$.

(b) $v(q)$ is a multiplicative function of $q$.

(c) If $p$ is a prime, $v(p)=2 p-1$.

Let $V(q)=\left(v(q) / q^{2}\right)^{3}$. Then $V(q)$ is also multiplicative. Note that

$$
\sum_{1 \leqq h \leqq q,(h, q)=1} e^{-2 \pi i(h / q) N}=C_{q}(N)=\sum_{d|N, d| q} \mu\left(\frac{q}{d}\right) d
$$

where $C_{q}(N)$ is Ramanujan's sum. Collecting these results we can write

$$
S(N)=\sum_{d \mid N} d \sum_{q=1, d \mid q}^{\infty} \mu\left(\frac{q}{d}\right) V(q)=\sum_{d \mid N} d \sum_{a=1}^{\infty} \mu(a) V(a d) .
$$

We state two well known results for multiplicative functions.

For any multiplicative function $f(q)$ where $f(p) \neq 1$ let

$$
F(d)=\sum_{a=1}^{\infty} \mu(a) f(a d) \text {. }
$$

Then if $p \nmid d, F\left(d p^{m}\right)=F(d)\left[f\left(p^{m}\right)-f\left(p^{m-1}\right) /(1-f(p))\right]$. Also

$$
\sum_{a=1}^{\infty} \mu(a) V(a)=\prod_{p}(1-V(p))=A .
$$

Here $A=\Pi_{p}\left(1-\left\{1-(1-1 / p)^{2}\right\}^{8}\right)$ where $A$ can be readily shown to be positive and less than one. Applying these results to the singular series, we have

THEOREM 4.

$$
S(N)=\prod_{p}(1-V(p)) \sum_{d|N|} d_{\left.p^{m} \mid d, p^{m+1}\right\}_{d}}\left(\frac{V\left(p^{m}\right)-V\left(p^{m+1}\right)}{1-V(p)}\right) .
$$

6. Generalizations. The first generalization to be considered is the case of $k n$-tuple products, $k \geqq 3, n \geqq 2$. Note that the method used in $\S 3$ on the minor arcs does not work for $k=2$.

In the general case we have 


$$
S(n, N, t)=S(t)=\sum_{x_{1} \cdots x_{n} \leqq N} e^{2 \pi i x_{1} x_{2} \cdots x_{n} t},
$$

and $R(n, k, N)=R(N)=$ number of solutions of $N=x_{11} \cdots x_{1 n}+x_{21} \cdots x_{2 n}$ $+\cdots+x_{k 1} \cdots x_{k n}$ where $R(N)=\int_{0}^{1} S^{k}(t) e^{-2 \pi i N t} d t$.

The Farey dissection of order $Q$ is as follows. Let $P=N^{1 / n}, Q=P^{n-1+c}$, $0<c<1, c$ to be assigned. For the major arcs let $q$ run from 1 to $P^{1-c}$. Define $S_{j}=\sum e^{2 \pi i x_{1} x_{2} \cdots x_{n} t}$ where the summation is over $x_{1} \cdots x_{n} \leqq N$, subject to conditions that the last $j$ of the $x$ 's are greater than $P$. Then we decompose $S(t)$ as follows: $S(t)=S_{0}(t)+C_{n, 1} S_{1}(t)-C_{n, 2} S_{2}(t) \cdots(-1)^{n} C_{n, n-1} S_{n-1}(t)$. As in $\S 3$ we show that $\max _{t \text { on } E}|S(t)|=O\left(P^{n-1+c+c}\right)$ and $\int_{E} S^{k}(t) e^{-2 \pi i N t} d t$ $<(\max |S(t)|)^{k-2} \int_{0}^{1}|S(t)|^{2} d t=O\left(P^{(n-1+c)(k-2)+n+\epsilon}\right)$. On a given $M_{h q}$ we split up $x_{j}=y_{j}+q_{j} z_{j}$ as before. Let

$$
v_{n-1}(q)=\sum_{1 \leqq y_{j} \leqq q ; j=1,2, \cdots, n-1 ; q \mid y_{1} y_{2} \cdots y_{n-1}} 1 .
$$

Define $\sum_{j}$ to indicate summation over $y_{1}, \cdots, y_{n-1}, z_{1}, \cdots, z_{n-1}$ subject to the conditions $q \mid y_{1} \cdots y_{n-1}$ and $x_{1} \cdots x_{n-1} \leqq P^{n-1}, x_{n-m}>P, m=0, \cdots, j$. Let

$$
\begin{gathered}
\sum^{\prime}=C_{n, 1} \sum_{1}-C_{n, 2} \sum_{2} \cdots(-1)^{n} C_{n, n-1} \sum_{n-1} \\
\sum^{\prime \prime}=\sum_{0}-\sum^{\prime} .
\end{gathered}
$$

Let

$$
T_{1}=\sum^{\prime} \frac{e^{2 \pi i \sigma N}-1}{2 \pi i g a}, \quad T_{2}=\sum^{\prime \prime} \frac{e^{2 \pi i g a P}-1}{2 \pi i g a}
$$

With this notation results analogous to those of $\$ 4$ follow, namely,

$$
S(t)=T_{1}+T_{2}+O\left(q P^{n-1}(\log N)^{n-2}\right),
$$

and

$$
\begin{aligned}
\sum_{1 \leqq h \leqq q \leqq P^{1-c},(h, q)=1} H_{h q}= & \sum_{h, q} \int_{-1 / q t}^{1 / q t}\left(T_{1}+T_{2}\right)^{k} e^{-2 \pi i o N} d g \\
& +O\left(P^{n(k-1)+2-8 c}(\log N)^{(n-1) k-1}\right) .
\end{aligned}
$$

The range of integration on each $M_{h q}$ can be extended to $(-\infty,+\infty)$ with an error term in $R(N)$ of $O\left(P^{(n-1+c)(k-2)+n+\epsilon)}\right.$. This will be the same order as the error term for minor arcs when $c=k /(k+1)$, thus making these error terms $O\left(P^{n(k-1)-(k-2) /(k+1)+\varepsilon}\right)$. We have

$$
\int_{-\infty}^{\infty}\left(\frac{e^{2 \pi i g N}-1}{2 \pi i g}\right)^{k} e^{-2 \pi i g N} d g=\frac{N^{k-1}}{(k-1) !} .
$$


Expanding $\left(T_{1}+T_{2}\right)^{k}$ and integrating we have $I_{0}=\left(\sum^{\prime} 1 / a\right)^{k} N^{k-1} /(k-1)$ ! with the other terms of order $N^{k-1}(\log N)^{(n-1) k-1} / q^{k-\epsilon}$. Thus we have

$$
\begin{aligned}
R(N)= & \sum_{1 \leqq q \leqq p^{1 / k+1}} \sum_{1 \leqq h \leqq q,(h, q)=1} e^{-2 \pi i(h / q) N}\left(\sum^{\prime} \frac{1}{a}\right)^{k} \frac{N^{k-1}}{(k-1) !} \\
& +O\left(N^{k-1}(\log N)^{(n-1) k-1}\right) .
\end{aligned}
$$

The summation over $q$ can be extended to $+\infty$ with an error of $O\left(P^{-\left((k-2-\epsilon)^{\prime}(k+1)\right)}\right)$ where $k-2-\epsilon>0$ requires $k>2$. Sharper estimates could probably overcome the restriction on $k$ here, but it seems much harder to eliminate the restriction in the consideration of the minor arcs.

Since

$$
\sum^{\prime} \frac{1}{a}=\frac{v_{n-1}(q)}{q^{n-1}} \frac{(\log N)^{n-1}}{(n-1) !}+O\left(\frac{\log ^{n-2} N}{q^{1-\epsilon}}\right),
$$

our singular series becomes

$$
\Im(n, k, N)=\sum_{1 \leqq q<\infty} \sum_{(h, q)=1,1 \leqq h \leqq q} e^{-2 \pi i(h / q) N} V(n, k, q)
$$

where $V(n, h, q)=V(q)=\left(v_{n-1}(q) / q^{n-1}\right)^{k}$.

We have the following: $v_{1}(q)=1 . v_{m}(q)=\sum_{1 \leqq y \leqq q}(y, q)^{m-1} v_{m-1}(q /(y, q))$ if $m>1$. Since $v_{m}(q)$ is multiplicative, $V(n, k, q)$ is also. In addition $v_{m}(p)=p^{m}-(p-1)^{m}$.

Then as before

$$
\Im\left(n_{b} k, N\right)=\prod_{p}(1-V(q)) \sum_{d \mid N} d \prod_{\left.p^{m} \mid d, p^{m+1}\right\}_{d}} \frac{V\left(p^{m}\right)-V\left(p^{m+1}\right)}{1-V(p)} .
$$

It is interesting to check this general result for the special case $n=2$ considered by Estermann $[2 ; 3]$. When $n=2, V(2, k, p)=p^{-k}$ and $\prod_{p}\left(1-p^{-k}\right)$ $=1 / \zeta(k) . V\left(2, k, p^{m}\right)=p^{-m k}$. Thus

$$
\begin{aligned}
S(2, k, N) & =\frac{1}{\zeta(k)} \sum_{d \mid N} d \prod_{p^{m} \mid d, p^{m+1}\{d}\left(\frac{p^{-m k}-p^{-(m+1) k}}{1-p^{-k}}\right) \\
& =\frac{1}{\zeta(k)} \sum_{d \mid N} d \prod_{p^{m} \mid d} p^{-m k}=\frac{1}{\zeta(k)} \sum_{d \mid N} d^{1-k}=\frac{1}{\zeta(k)} \sigma_{1-k}(N)
\end{aligned}
$$

where $\sigma_{r}(N)=$ the sum of the $r^{t h}$ powers of the divisors of $N$. This checks with Estermann's leading terms.

We can write the singular series in the form

$\mathfrak{S}_{n, k}(N)=\prod_{p\{N}(1-V(p)) \sum_{d \mid N} d \prod_{\left.p^{m} \mid d, p^{m+1}\right\} d}\left(\left\{1-V\left(p^{m+1}\right)\right\}-\left\{1-V\left(p^{m}\right)\right\}\right)$.

The factor $1-V(p)=1-\left\{1-(1-1 / p)^{n-1}\right\}^{k}$, for $p \nmid N$, in a suitably defined sense gives the probability that $p$ does not divide the greatest common di- 
visor of the $k$ products making up $N$. For $p \mid N$ the factor $\left\{1-V\left(p^{m+1}\right)\right\}$ $-\left\{1-V\left(p^{m}\right)\right\}$ gives the probability that $p^{m}$ divides the g.c.d. of the $k$ products while $p^{m+1}$ does not. This allows for the possibility that $d$ could divide each $n$-tuple if $d \mid N$.

A further generalization leads immediately to the case where the $j$ th product has $m_{j}$ factors. For example, we can study representations of the form $N=x_{11} x_{12}+x_{21} x_{22} x_{23}+x_{31} x_{32} x_{33} x_{34}$.

If we let $n=\max _{j} n_{j}$ then consider a Farey dissection of order $Q=P^{n-1+c}$ where $P=N^{1 / n}$ and $c=k /(k+1)$. Then the error terms are the same order as in $\S 6$. Each $S\left(n_{j}, t\right)$ is estimated separately and we finally get

$$
R(N)=\Im(N) \prod_{j} \frac{(\log N)^{n_{j}-1}}{\left(n_{j}-1\right) !} \frac{N^{k-1}}{(k-1) !}\left(1+O\left(\frac{1}{\log N}\right)\right) .
$$

Let

$$
V\left(n_{1}, \cdots, n_{k}, q\right)=V(q)=\prod_{j}\left(\frac{v_{n_{j}-1}(q)}{q^{n_{j}-1}}\right)
$$

Then

$$
\Im\left(n_{1}, \cdots, n_{k}, N\right)=\prod_{p \nmid N}\left((1-V(p)) \sum_{d \mid N} \prod_{\left.p^{m} \mid d, p^{m+1}\right\} d}\left\{V\left(p^{m}\right)-V\left(p^{m+1}\right)\right\}\right.
$$

and the previous probability interpretation of these factors carries over.

\section{BIBLIOGRAPHY}

1. R. Bellman, On some divisor sums associated with diophantine equations, Quart. J. Math. Oxford Ser. (2) vol. 1 (1950) pp. 136-146.

2. T. Estermann, On the representations of a number as the sum of three products, Proc. London Math. Soc. (2) vol. 29 (1929) pp. 453-478.

3. - On the representations of a number as the sum of two products, Proc. London Math. Soc. (2) vol. 31 (1930) pp. 123-133.

4. G. H. Hardy and E. M. Wright, An introduction to the theory of numbers, Oxford, 1938.

5. A. E. Ingham, Some asymptotic formulae in the theory of numbers, J. London Math. Soc. vol. 2 (1927) pp. 207-208.

6. E. Landau, Vorlesungen ïber Zahlentheorie, New York, 1947.

7. E. C. Titchmarsh, Some problems in the analytic theory of numbers, Quart. J. Math. Oxford Ser. vol. 13 (1942).

UNIVERSITY OF ILLINOIS,

URBANA, ILL.

The Rand Corporation,

Santa Monica, Calif. 